\title{
RIGIDITY OF CYLINDERS WITHOUT CONJUGATE POINTS*
}

\author{
HENRIK KOEHLER ${ }^{\dagger}$
}

\begin{abstract}
During the last decades, several investigations were concerned with rigidity statements for manifolds without conjugate points (some results can be found in the references). Based on an idea by E.Hopf $[\mathrm{H}]$, K.Burns and G.Knieper proved in [BK] that cylinders without conjugate points and with a lower sectional curvature bound must be flat if the length of the shortest loop at every point is globally bounded.

The present article reduces the last condition to a limit for the asymptotic growth of loop-length as the basepoint approaches the ends of the cylinder (Thm. 18). Along the way, the shape of cylinders without conjugate points is characterized: The loop-length must be strictly monotone increasing to both ends outside a - possibly empty - tube consisting of closed geodesics (Thm. 10).
\end{abstract}

Key words. Global Riemannian geometry, rigidity results, curvature bounds

AMS subject classifications. 53C21, 53C24

\section{Preliminaries.}

1.1. Conjugate points, Riccati equation. Let $M$ be a smooth, complete surface with a Riemannian metric $\langle$,$\rangle and sectional curvature K$; furthermore $T M$ the tangent bundle and $\pi: T M \underset{\sim}{\longrightarrow}$ the footpoint-projection, $\widetilde{M}$ the universal Riemannian covering of $M$ and $\widetilde{\pi}: \widetilde{M} \rightarrow M$ the projection.

Given $X \subseteq M$ note by $S X:=\{v \in T M \mid \pi(v) \in X ;\|v\|=1\}$ the unit vectors with footpoint in $X$; let $\lambda$ for every $p$ denote the Lebesgue-measure on $S_{p} M, \mu=\operatorname{vol}_{M} \times \lambda$ the Liouville-measure on $S M$ and $g^{t}: S M \rightarrow S M,\left.v \mapsto \frac{d}{d s}\right|_{s=t} \exp _{\pi(v)}(s v)$ the geodesic flow at time $t$.

For $v \in S M$ regard the geodesic $\gamma_{v}(t):=\exp _{\pi(v)}(t v)$, parameterized by arclength, with sectional curvature $K(t):=K\left(\gamma_{v}(t)\right)$; the Jacobi equation related to $\gamma_{v}$ is then

$$
y^{\prime \prime}(t)+K(t) y(t)=0 .
$$

Definition 1. $M$ is called without conjugate points, if for any $v \in S M$, every non-trivial solution of $\left(J_{v}\right)$ vanishes once at most.

If $M$ is a surface without conjugate points, then for all $v \in S M, s \in \mathbb{R}$ there exists a solution $y(v, s, t)$ of $\left(J_{v}\right)$ with boundary values $y(v, s, 0)=1$ and $y(v, s, s)=0$. The next theorem characterizes this property (for the 3rd part, see $[\mathrm{H}]$ ).

THEOREM 2. The following criteria are equivalent:

1. $M$ has no conjugate points;

2. any two geodesics in $\widetilde{M}$ can intersect once at most, in particular all geodesics in $\widetilde{M}$ are minimal;

3. the stable resp. unstable solutions of $\left(J_{v}\right)$, defined by $y_{-}(v, t):=\lim _{s \rightarrow \infty} y(v, s, t)$ and $y_{+}(v, t):=\lim _{s \rightarrow-\infty} y(v, s, t)$ respectively, exist $\forall v \in S M$ on the entire $\mathbb{R}$.

\footnotetext{
* Received October 26, 2006; accepted for publication March 29, 2007.

$\dagger$ Fakultät für Mathematik, Ruhr-Universität Bochum, D-44780 Bochum, Germany (henrik. koehler@rub.de).
} 
The Riccati equation related to $\gamma_{v}$ is

$$
u^{\prime}(t)+u^{2}(t)+K(t)=0 ;
$$

it is obtained from $\left(J_{v}\right)$ by transformation $u=y^{\prime} / y$. In general, the zero locus of $y$ must be excepted. In absence of conjugate points $y_{ \pm}>0$, and $u_{-}(v, t):=\frac{y_{-}^{\prime}(v, t)}{y-(v, t)}$ and $u_{+}(v, t):=\frac{y_{+}^{\prime}(v, t)}{y_{+}(v, t)}$ solve $\left(R_{v}\right)$ on $\mathbb{R}$ for every $v \in S M$. Set $U(v):=u_{+}(v, 0)$.

1.2. Comparison theorems. The existence of a lower curvature boundary allows us to compare $M$ with surfaces of constant curvature:

Proposition 3. Suppose $M$ is free of conjugate points and $K \geq-b^{2}$ for some $b>0$. For $A, B, C \in M$ let $\triangle \subset M$ denote the triangle with vertices $A, B, C$, whereas the edges are minimal geodesic segments. If $M^{\prime}$ is the plane with constant curvature $K^{\prime}=-b^{2}$ and $\triangle^{\prime} \subset M^{\prime}$ is the geodesic triangle spanned by $A^{\prime}, B^{\prime}, C^{\prime}$, and if $d^{\prime}\left(A^{\prime}, C^{\prime}\right)=d(A, C), d^{\prime}\left(A^{\prime}, B^{\prime}\right)=d(A, B)$ and $\angle\left(C^{\prime} A^{\prime} B^{\prime}\right)=\angle(C A B)$, then $d^{\prime}\left(B^{\prime}, C^{\prime}\right) \geq d(B, C)$.

Proof. This is an application of a triangle-comparison-theorem.

Lemma 4. For $v \in S M, b>0$ and $r<s$ let $K(t) \geq-b^{2} \forall t \in[r, s]$. If $y$ is a solution of $\left(J_{v}\right)$ with $0<y(t) \forall t \in[r, s]$, then $y(t) \leq y(r) \cosh (b(t-r))+$ $y^{\prime}(r) \sinh (b(t-r)) / b$ and $\frac{y^{\prime}(t)}{y(t)}<b \operatorname{coth}(b(t-r))$ hold for all $t \in[r, s]$.

Proof. Set $z(t):=y(r) \cosh (b(t-r))+y^{\prime}(r) \sinh (b(t-r)) / b$ and $w(t):=y^{\prime}(t) z(t)-$ $y(t) z^{\prime}(t)$; remark, that $z$ cannot vanish twice and $w(r)=0$. Also set $\hat{s}:=\sup \{t \in$ $[r, s] \mid z(t)>0\}$; then for all $t \in[r, \hat{s}]$

$$
\begin{aligned}
w^{\prime}(t) & =y^{\prime \prime}(t) z(t)-y(t) z^{\prime \prime}(t)=\left(-K(t)-b^{2}\right) y(t) z(t) \leq 0 \\
\Rightarrow \quad w(t) & =\int_{r}^{t} w^{\prime}(u) d u \leq 0 \Rightarrow \frac{w(t)}{y(t) z(t)}=\frac{y^{\prime}(t)}{y(t)}-\frac{z^{\prime}(t)}{z(t)} \leq 0 \\
\Rightarrow \quad y(t) & =y(r) \exp \left(\int_{r}^{t} \frac{y^{\prime}(u) d u}{y(u)}\right) \leq y(r) \exp \left(\int_{r}^{t} \frac{z^{\prime}(u) d u}{z(u)}\right)=z(t) .
\end{aligned}
$$

Therefore $\hat{s}=s$, as otherwise $0<y(\hat{s}) \leq z(\hat{s})=0$. The second inequality now results from

$$
\frac{y^{\prime}(t)}{y(t)} \leq \frac{z^{\prime}(t)}{z(t)}=\frac{b y(r) \sinh (b(t-r))+y^{\prime}(r) \cosh (b(t-r))}{y(r) \cosh (b(t-r))+y^{\prime}(r) \sinh (b(t-r)) / b}<b \operatorname{coth}(b(t-r)) .
$$

Using a similar method, Hopf $[\mathrm{H}]$ showed:

Corollary 5. Let $M$ be free of conjugate points, then $U$ is $\mu$-measurable. If in addition there is $a b>0$ with $K \geq-b^{2}$, then $|U| \leq b$.

The flatness-condition is mainly based on $[\mathrm{BK}]$, Lem. 1.3:

Lemma 6. Let $M$ without conjugate points and $Q$ a compact subset of $M$ with $\partial Q$ piecewise smooth. Then

$$
\int_{S Q} U^{2}(v) d \mu(v) \leq-2 \pi \int_{Q} K(p) d v o_{M}(p)+2 \int_{\partial Q} \int_{S_{p} M}|U(v)| d \lambda(v) d L(p) .
$$




\section{Cylinders.}

2.1. Geodesic loops $\&$ closed geodesics. Consider a smooth cylinder $C$ (i.e. a complete surface diffeomorphic to $\mathbb{R} \times S^{1}$ ) equipped with a Riemannian metric $\langle\cdot, \cdot\rangle$ without conjugate points and curvature $K$. Denote by $\widetilde{C} \simeq \mathbb{R}^{2}$ the universal Riemannian covering for $C$. The fundamental group is $\pi_{1}(C) \simeq \mathbb{Z}$; let $\varphi: \widetilde{C} \rightarrow \widetilde{C}$ be a generator of the deck transformation group of $C$.

DEFINITION 7.

1. For $l>0$, an arclength-parameterized geodesic segment $c:[0, l] \rightarrow C$ with $c(0)=c(l)$ is called geodesic loop with basepoint $c(0)$.

2. If further $c^{\prime}(0)=c^{\prime}(l)$ (and so $c(t+l)=c(t) \forall t$ ), $c$ is a closed geodesic.

Remark that closed geodesics cannot have transversal self-intersections: If $c(u)=$ $c(v)$ for some $u<v \in[0, l]$ and $\widetilde{c}: \mathbb{R} \rightarrow \widetilde{C}$ denotes a lift of $c$, there would be $m, z \in \mathbb{Z} \backslash\{0\}$ such that $\varphi^{z} \widetilde{c}(t)=\widetilde{c}(t+l) \forall t$ and $\widetilde{c}(v)=\varphi^{m} \widetilde{c}(u)$

$$
\begin{aligned}
& \Rightarrow \widetilde{c}(v+n l)=\varphi^{n z} \widetilde{c}(v)=\varphi^{n z+m} \widetilde{c}(u)=\varphi^{m} \widetilde{c}(u+n l) \forall n \in \mathbb{Z} \\
& \Rightarrow \widetilde{c}(t+v-u)=\varphi^{m} \widetilde{c}(t) \forall t \in \mathbb{R}
\end{aligned}
$$

by Thm. 2 ; hence $c(t+v-u)=c(t) \forall t$. Therefore we may always assume closed geodesics to be simple, for $l$ should be the (least) period of $c$.

Proposition 8. A geodesic loop is a closed geodesic, iff it has minimal length in the set of non-contractible loops in $C$.

Proof. Take $c:[0 ; l] \rightarrow C$ to be a simple geodesic loop of length $l$. If $c$ is minimal, $c^{\prime}(l)=c^{\prime}(0)$; as it could be shortened by variation if it would contain a vertex at $c(0)$.

On the other hand, if $c$ is a closed geodesic, let $\sigma: \mathbb{R} \rightarrow \widetilde{C}$ be a lift; w.l.o.g. suppose $\sigma(l)=\varphi \sigma(0)$. Also take an arbitrary non-contractible loop $a:[0 ; \lambda] \rightarrow C$ of length $\lambda$ with a lift $\alpha:[0 ; \lambda] \rightarrow \widetilde{C}$. Then $\alpha(\lambda)=\varphi^{z} \alpha(0)$ for some $z \in \mathbb{Z} \backslash\{0\}$.

As $\varphi$ operates isometrically on $\widetilde{C}$, the triangle-inequality implies

$$
\begin{aligned}
n l|z| & =d(\sigma(0), \sigma(n z l)) \\
& \leq d(\sigma(0), \alpha(0))+d\left(\varphi^{n z} \alpha(0), \varphi^{n z} \sigma(0)\right)+\sum_{j=0}^{n-1} d\left(\varphi^{j z} \alpha(0), \varphi^{(j+1) z} \alpha(0)\right) \\
& =2 d(\sigma(0), \alpha(0))+\sum_{j=0}^{n-1} d\left(\varphi^{j} \alpha(0), \varphi^{j} \alpha(\lambda)\right) \\
& =2 d(\sigma(0), \alpha(0))+n d(\alpha(0), \alpha(\lambda)) \leq 2 d(\sigma(0), \alpha(0))+n \lambda \quad \forall n \in \mathbb{N},
\end{aligned}
$$

which proves $\lambda \geq|z| l \geq l$ as $n \rightarrow \infty$.

Let $\gamma: \mathbb{R} \rightarrow C$ be an arclength-parameterized geodesic s.th. $C \backslash \gamma$ is simplyconnected, with $\gamma_{1}$ a lift to $\widetilde{C}$ and $\gamma_{2}=\varphi \gamma_{1} \cdot \gamma_{1}$ and $\gamma_{2}$ cannot intersect, because then $\gamma$ would contain self-intersections and $C \backslash \gamma$ could not be connected.

Set $l(s):=d\left(\gamma_{1}(s), \gamma_{2}(s)\right)$ and denote by $\sigma_{s}$ the arclength-parameterized geodesic through $\sigma_{s}(0)=\gamma_{1}(s)$ and $\sigma_{s}(l(s))=\gamma_{2}(s)$ and by $c_{s}:=\widetilde{\pi} \circ \sigma_{s}$ the projection of $\sigma_{s}$ onto $C$; then $c_{s} \mid[0 ; l(s)]$ is a geodesic loop with basepoint $\gamma(s)$.

Let $\alpha_{s}:=\angle\left(\sigma_{s}^{\prime}(0), \gamma_{1}^{\prime}(s)\right)$ and $\beta_{s}:=\angle\left(\gamma_{2}^{\prime}(s),-\sigma_{s}^{\prime}(l(s))\right)$ denote the angles between $\gamma_{1}$ resp. $\gamma_{2}$ and $\sigma_{s}$; obviously $0<\alpha_{s}, \beta_{s}<\pi \quad \forall s \in \mathbb{R}$. 


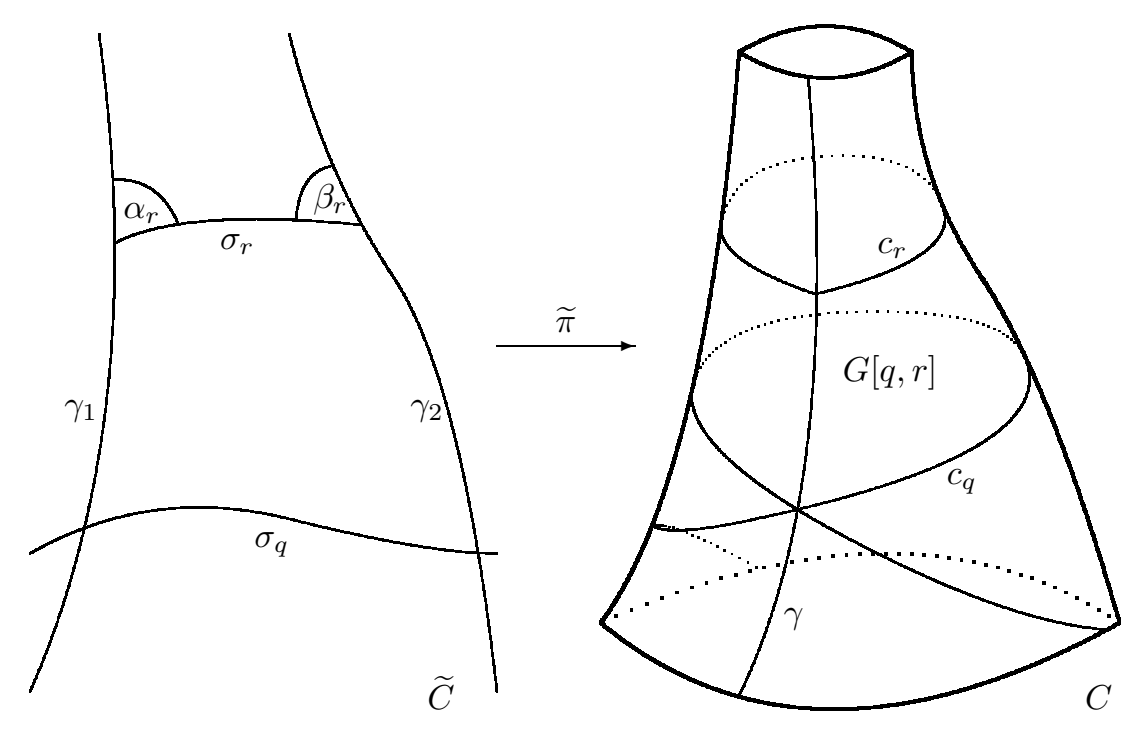

FIG. 1. Geodesic loops and their liftings

For each interval $I=[q, r]$ or $I=] q, r[\subseteq \mathbb{R}$ with $-\infty \leq q \leq r \leq \infty$ define $G I:=\left\{c_{s}(t) \mid s \in I, t \in[0 ; l(s)]\right\} \subseteq C$.

For fixed $s$ consider the geodesic variation

$$
H:] s-\varepsilon, s+\varepsilon\left[\times[0 ; l(s)] \rightarrow \widetilde{C}, H(r, t)=\sigma_{r}(t l(r) / l(s)) .\right.
$$

The related Jacobi-vectorfield is $Y_{s}(t):=\left.\frac{\partial}{\partial r}\right|_{r=s} H(r, t)$, and its normal component $y_{s}(t):=\left\|Y_{s}(t)-\left\langle Y_{s}(t), \sigma_{s}^{\prime}(t)\right\rangle \sigma_{s}^{\prime}(t)\right\|$. $y_{s}$ is strictly positive since it could vanish at most for a single $t \in[0, l(s)]$ - while $y_{s}(0)=\sin \alpha_{s}(t)>0$ and $y_{s}(l(s))=\sin \beta_{s}(t)>0$.

The 1 st variation formula claims

$$
l^{\prime}(s)=\left.\left\langle Y_{s}(t), \sigma_{s}^{\prime}(t)\right\rangle\right|_{0} ^{l(s)}=-\cos \beta_{s}-\cos \alpha_{s}=-2 \cos \frac{\alpha_{s}-\beta_{s}}{2} \cos \frac{\alpha_{s}+\beta_{s}}{2} .
$$

REMARK 9. Because of $-\pi<\alpha_{s}-\beta_{s}<\pi$, the following holds: $l^{\prime}(s)=0 \Leftrightarrow \alpha_{s}+\beta_{s}=\pi \Leftrightarrow c_{s}^{\prime}(l(s))=c_{s}^{\prime}(0) \Leftrightarrow c_{s}$ is a closed geodesic.

TheOrem 10. There exist $-\infty \leq q \leq r \leq \infty$, such that all geodesic loops in $G[q, r]$ are closed geodesics of constant length $l \equiv l(q)$, and $l^{\prime}(s)>0$ for $\left.s \in\right] r, \infty[$ and $l^{\prime}(s)<0$ for $\left.s \in\right]-\infty, q[$.

Proof. Since $C \backslash \gamma$ is contractible, every closed geodesic must be intersected by $\gamma$ in some point and is thus a loop to this basepoint.

If there don't exist any closed geodesics, $l^{\prime}$ has the same sign everywhere according to Rem. 9; in this case set $q=r= \pm \infty$ depending on whether $l^{\prime}<0$ or $l^{\prime}>0$.

In the other case, take $c_{a}$ and $c_{b}$ to be closed geodesics for some $a \leq b$. Due to Prop. $8, l(a) \leq l(b) \leq l(a) \Rightarrow l(a)=l(b)$.

Furthermore $l \geq l(a)$ on the entire $\mathbb{R}$; let $m \in[a, b]$ be a maximum locus for $l$ on $[a, b]$. Then $l^{\prime}(m)=0$, i.e. $c_{m}$ is a closed geodesic. Thus the same argument states 
$l(m)=l(a)$, so $l$ must be constant on $[a, b]$. As $l^{\prime} \equiv 0$ on $[a, b]$, all geodesic loops $G\{s\}$ with $s \in[a, b]$ are closed geodesics.

The claim now follows by setting $q:=\inf \left\{s \in \mathbb{R} \mid l^{\prime}(s)=0\right\}$ and $r:=\sup \{s \in$ $\left.\mathbb{R} \mid l^{\prime}(s)=0\right\}$.
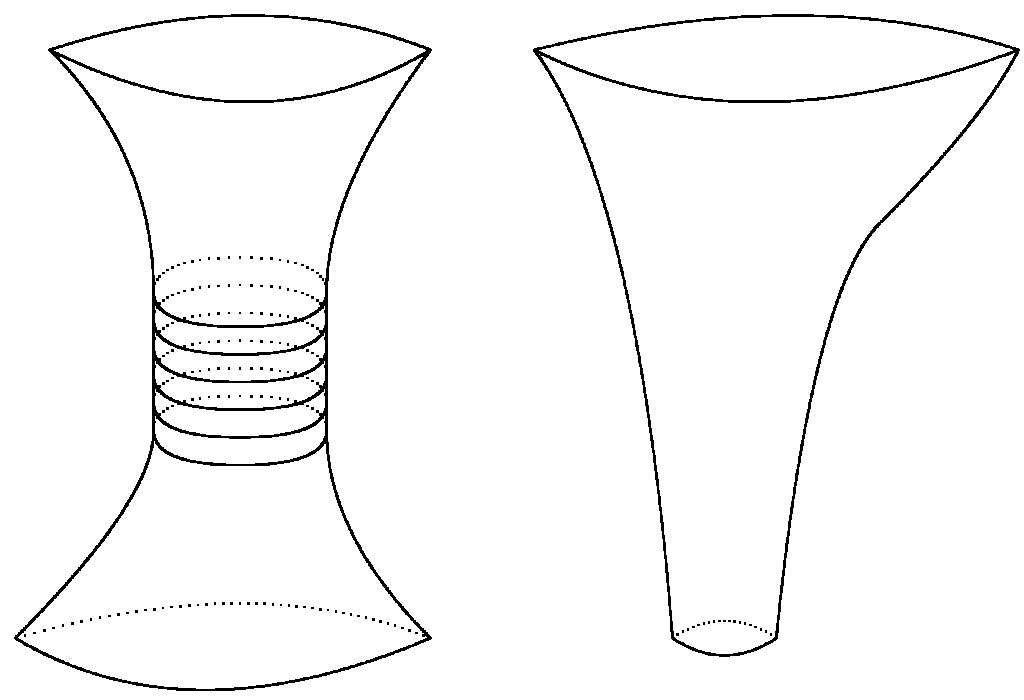

FIG. 2. Two types of cylinders: with and without closed geodesics

REMARK 11. Thm. 10 provides a classification of cylinders without conjugate points in types with resp. without closed geodesics.

To simplify the notation, mainly in section 2.3 , assume that the choice of the parameterization for $\gamma$ complies with either of these conditions:

1. If $C$ possesses closed geodesics, $c_{0}$ shall be one of them. This effects $l^{\prime} \leq 0$ on $\mathbb{R}_{-}$and $l^{\prime} \geq 0$ on $\mathbb{R}_{+}$.

2. If $C$ doesn't contain closed geodesics, suppose that $l^{\prime}>0$ everywhere.

\subsection{An integral inequality for $U^{2}$.}

\section{LEMMA 12.}

1. The geodesic $\gamma$ can be chosen minimal in $C$.

2. If $\liminf _{s \rightarrow \pm \infty} l(s) /|s|<2$ then $G \mathbb{R}=C$.

Proof. The 1st claim is proved in [BK], p. 630. For the 2nd part, suppose that there exists some $p \in C \backslash G \mathbb{R}$ with lift $\widetilde{p} \in \widetilde{C}$, which is w.l.o.g. situated in the half-strip between $\gamma_{1}\left|\mathbb{R}_{+}, \gamma_{2}\right| \mathbb{R}_{+}$and $\sigma_{0}$. Let $\psi_{1}$ and $\psi_{2} \subset \widetilde{C}$ be the geodesic segments from $\widetilde{p}$ to $\gamma_{1}(0)$ and $\gamma_{2}(0)$ respectively. $\sigma_{s}$ varies continuously in $s$, thus (as it does near $s=0)$ for every $s \geq 0$ it intersects $\psi_{1}$ in some point $p_{1}(s)$ and $\psi_{2}$ in another point $p_{2}(s)$. 
The triangle-inequality states

$$
\begin{aligned}
2 s & =d\left(\gamma_{1}(0), \gamma_{1}(s)\right)+d\left(\gamma_{2}(0), \gamma_{2}(s)\right) \\
& \leq d\left(\gamma_{1}(0), p_{1}(s)\right)+d\left(p_{1}(s), \gamma_{1}(s)\right)+d\left(\gamma_{2}(0), p_{2}(s)\right)+d\left(p_{2}(s), \gamma_{2}(s)\right) \\
& \leq L\left(\psi_{1}\right)+L\left(\psi_{2}\right)+l(s) \\
\Rightarrow 2 & \leq \liminf _{s \rightarrow \infty} l(s) / s
\end{aligned}
$$

- the 2nd claim is just the negation.

REMARK 13. For every $s \in \mathbb{R}, t \in[0, l(s)], y_{s}(t)$ is the density of the Riemannian volume with respect to the product measure of the length on $c_{s}$ and that on $\gamma$.

To prove this, set $\partial_{s}(s, t):=\frac{\partial}{\partial s} \sigma_{s}(t)$ and $\partial_{t}(s, t):=\frac{\partial}{\partial t} \sigma_{s}(t)=\sigma_{s}^{\prime}(t)$. Using $\left\|\sigma_{s}^{\prime}(t)\right\| \equiv 1$ and $\frac{\partial}{\partial s} \sigma_{s}(t)=Y_{s}(t)-\sigma_{s}^{\prime}(t) l^{\prime}(s) t / l(s)$ compute

$$
\begin{aligned}
\frac{d^{2} v_{C}(s, t)}{d s d t} & =\sqrt{\operatorname{det}\left(\begin{array}{cc}
\left\langle\partial_{s}, \partial_{s}\right\rangle & \left\langle\partial_{s}, \partial_{t}\right\rangle \\
\left\langle\partial_{t}, \partial_{s}\right\rangle & \left\langle\partial_{t}, \partial_{t}\right\rangle
\end{array}\right)(s, t)} \\
& =\left\|\partial_{s}(s, t)-\left\langle\partial_{s}(s, t), \sigma_{s}^{\prime}(t)\right\rangle \sigma_{s}^{\prime}(t)\right\| \\
& =\left\|Y_{s}(t)-\left\langle Y_{s}(t), \sigma_{s}^{\prime}(t)\right\rangle \sigma_{s}^{\prime}(t)\right\|=y_{s}(t) .
\end{aligned}
$$

In the sequel, abbreviate

$$
V(s):=\int_{0}^{l(s)} \int_{S c_{s}(t)} U^{2}(v) d \lambda(v) y_{s}(t) d t \geq 0
$$

LEMMA 14. For fixed $q<r \in \mathbb{R}$

$$
\left(\int_{q}^{r} V(s) d s\right)^{2} \leq 32 \pi\left(V(q) \int_{0}^{l(q)} \frac{d t}{y_{q}(t)}+V(r) \int_{0}^{l(r)} \frac{d t}{y_{r}(t)}\right)+8 \pi^{2}\left(\alpha_{r}+\beta_{r}-\alpha_{q}-\beta_{q}\right)^{2}
$$

Proof. Lem. 6 gives

$$
\begin{aligned}
\int_{S G[q, r]} U^{2}(v) d \mu(v) \leq & -2 \pi \int_{G[q, r]} K(p) d \operatorname{vol}_{C}(p)+2 \int_{0}^{l(q)} \int_{S c_{q}(t)}|U(v)| d \lambda(v) d t \\
& +2 \int_{0}^{l(r)} \int_{S c_{r}(t)}|U(v)| d \lambda(v) d t
\end{aligned}
$$

wherein the curvature-integral is

$$
\int_{G[q, r]} K(p) d \operatorname{vol}_{C}(p)=\alpha_{q}+\beta_{q}-\alpha_{r}-\beta_{r}
$$

due to Gauss-Bonnet. Applying the Cauchy-Schwarz-inequality twice, the other integrals can be estimated by

$$
\begin{aligned}
\left(\int_{0}^{l(s)} \int_{S c_{s}(t)}|U(v)| d \lambda(v) d t\right)^{2} & \leq \int_{0}^{l(s)}\left(\int_{S c_{s}(t)}|U(v)| d \lambda(v)\right)^{2} y_{s}(t) d t \int_{0}^{l(s)} \frac{d t}{y_{s}(t)} \\
& \leq \int_{0}^{l(s)} 2 \pi \int_{S c_{s}(t)} U^{2}(v) d \lambda(v) y_{s}(t) d t \int_{0}^{l(s)} \frac{d t}{y_{s}(t)} \\
& =2 \pi V(s) \int_{0}^{l(s)} \frac{d t}{y_{s}(t)}
\end{aligned}
$$


On the other hand, Rem. 13 allows to write

$$
\int_{S G[q, r]} U^{2}(v) d \mu(v)=\int_{q}^{r} \int_{0}^{l(s)} \int_{S c_{s}(t)} U^{2}(v) d \lambda(v) y_{s}(t) d t d s=\int_{q}^{r} V(s) d s .
$$

The ineqs. (1) to (4) gather to

$$
\begin{aligned}
\int_{q}^{r} V(s) d s \leq & \sqrt{8 \pi V(q) \int_{0}^{l(q)} \frac{d t}{y_{q}(t)}}+\sqrt{8 \pi V(r) \int_{0}^{l(r)} \frac{d t}{y_{r}(t)}} \\
& +2 \pi\left(\alpha_{r}+\beta_{r}-\alpha_{q}-\beta_{q}\right) .
\end{aligned}
$$

Since $0 \leq(\sqrt{a}-\sqrt{c})^{2} \Rightarrow a+c+2 \sqrt{a c} \leq 2 a+2 c \Rightarrow \sqrt{a}+\sqrt{c} \leq \sqrt{2(a+c)}$ for arbitrary $a, c \geq 0$ the right-hand side of (5) can be estimated again by

$$
\begin{aligned}
& \sqrt{8 \pi V(q) \int_{0}^{l(q)} \frac{d t}{y_{q}(t)}}+\sqrt{8 \pi V(r) \int_{0}^{l(r)} \frac{d t}{y_{r}(t)}}+2 \pi\left(\alpha_{r}+\beta_{r}-\alpha_{q}-\beta_{q}\right) \\
\leq & \sqrt{16 \pi V(q) \int_{0}^{l(q)} \frac{d t}{y_{q}(t)}+16 \pi V(r) \int_{0}^{l(r)} \frac{d t}{y_{r}(t)}}+2 \pi\left(\alpha_{r}+\beta_{r}-\alpha_{q}-\beta_{q}\right) \\
\leq & \sqrt{32 \pi V(q) \int_{0}^{l(q)} \frac{d t}{y_{q}(t)}+32 \pi V(r) \int_{0}^{l(r)} \frac{d t}{y_{r}(t)}+8 \pi^{2}\left(\alpha_{r}+\beta_{r}-\alpha_{q}-\beta_{q}\right)^{2}},
\end{aligned}
$$

which leads to the claimed inequality.

2.3. Flatness condition in case of bounded curvature. During this section, suppose that $K>-b^{2}$ for some $b>0$ and that $\gamma$ is minimal (cf. Lem. 12).

Lemma 15. $\left|\cos \alpha_{s}\right|,\left|\cos \beta_{s}\right| \leq \tanh (b l(s) / 2) \quad \forall s \in \mathbb{R}$.

Proof. For every $r$, the geodesic segment from $\gamma_{2}(s)=\sigma_{s}(l(s))$ to $\gamma_{1}(s+r)$ is longer than $d_{C}(\gamma(s), \gamma(s+r))=r$, because it is a lift of a geodesic segment in $C$ between $\gamma(s)$ and $\gamma(s+r)$, and $\gamma$ is minimal.

In a plane of constant curvature $-b^{2}$, consider a geodesic triangle, where two edges, one of length $l(s)$ and one of length $r$, span an angle of $\alpha_{s}$. The length of the edge on the opposite side shall be $a$. Comparing this triangle with the geodesic triangle in $\widetilde{C}$ with vertices $\gamma_{1}(s), \gamma_{1}(s+r)$ and $\gamma_{2}(s)$, Prop. 3 implies $a \geq d\left(\gamma_{2}(s), \gamma_{1}(s+r)\right)>r$.

Hence the hyperbolic cosine-theorem holds for any $r>0$

$$
\begin{aligned}
\cos \alpha_{s} & =\frac{\cosh (b l(s)) \cosh (b r)-\cosh (b a)}{\sinh (b l(s)) \sinh (b r)}<\frac{(\cosh (b l(s))-1) \cosh (b r)}{\sinh (b l(s)) \sinh (b r)} \\
\Rightarrow \quad \cos \alpha_{s} & \leq \frac{\cosh (b l(s))-1}{\sinh (b l(s))}=\tanh (b l(s) / 2),
\end{aligned}
$$

as $r \rightarrow \infty$. The same estimation, applied to $\beta_{s}$ and the opponent angles $\pi-\alpha_{s}, \pi-\beta_{s}$ proves the claim.

Corollary 16. $\int_{0}^{l(s)} d t / y_{s}(t)<\frac{\pi}{b} \cosh ^{2}(b l(s) / 2) \quad \forall s \in \mathbb{R}$.

Proof. First, claim

$$
y_{s}(t) \geq x_{s}(t):=\frac{\cosh (b(t-l(s) / 2))}{\cosh ^{2}(b l(s) / 2)} \quad \forall s \in \mathbb{R}, t \in[0, l(s)] .
$$


In accordance with Lem. 15,

$$
y_{s}(0)=\sin \alpha_{s}=\sqrt{1-\cos ^{2} \alpha_{s}} \geq \sqrt{1-\tanh ^{2} \frac{b l(s)}{2}}=\frac{1}{\cosh (b l(s) / 2)}=x_{s}(0)
$$

$\forall s \in \mathbb{R}$ and as well $y_{s}(l(s)) \geq 1 / \cosh (b l(s) / 2)=x_{s}(l(s))$ (cf. [BK] Lem. 2.4). Fix $0<\delta<1$ and assume that there are $s, t$ s.th. $y_{s}(t)<\delta x_{s}(t)$. Then define $\tau:=\inf \{t \in$ $\left.[0, l(s)] \mid y_{s}(t)<\delta x_{s}(t)\right\}$; obviously $\tau>0, y_{s}(\tau)=\delta x_{s}(\tau)$ and $y_{s}^{\prime}(\tau) \leq \delta x_{s}^{\prime}(\tau)$. As $y_{s}>0$ on $[0, l(s)]$, by Lem. 4 get for $\tau \leq t \leq l(s)$

$$
\begin{aligned}
y_{s}(t) & \leq y_{s}(\tau) \cosh (b(t-\tau))+y_{s}^{\prime}(\tau) \frac{\sinh (b(t-\tau))}{b} \\
& \leq \delta x_{s}(\tau) \cosh (b(t-\tau))+\delta x_{s}^{\prime}(\tau) \frac{\sinh (b(t-\tau))}{b}=\delta x_{s}(t)
\end{aligned}
$$

- where the last equality refers to the fact, that both sides solve the Jacobi equation with $K \equiv-b^{2}$ and coincide in $\tau$ in their values and 1st derivatives.

But that leads to the contradiction $y_{s}(l(s)) \leq \delta x_{s}(l(s))<x_{s}(l(s)) \leq y_{s}(l(s))$, which shows $y_{s} \geq \delta x_{s}$. Since $\delta$ can be chosen arbitrarily, $y_{s} \geq \sup _{\delta<1} \delta x_{s}=x_{s}$.

Thus

$$
\begin{aligned}
\int_{0}^{l(s)} \frac{d t}{y_{s}(t)} & \leq \int_{0}^{l(s)} \frac{\cosh ^{2}(b l(s) / 2) d t}{\cosh (b(t-l(s) / 2))}=\int_{-l(s) / 2}^{l(s) / 2} \frac{\cosh ^{2}(b l(s) / 2) d t}{\cosh (b t)} \\
& =\cosh ^{2} \frac{b l(s)}{2} \int_{-l(s) / 2}^{l(s) / 2} \frac{2 \mathrm{e}^{b t} d t}{\mathrm{e}^{2 b t}+1}=\cosh ^{2} \frac{b l(s)}{2} \int_{\mathrm{e}^{-b l(s) / 2}}^{\mathrm{e}^{b l(s) / 2}} \frac{2 d x}{b\left(x^{2}+1\right)} \\
& =\left.\cosh ^{2}(b l(s) / 2) \frac{2 \arctan \left(\mathrm{e}^{b t}\right)}{b}\right|_{-l(s) / 2} ^{l(s) / 2}<\frac{\pi}{b} \cosh ^{2}(b l(s) / 2) .
\end{aligned}
$$

Lemma 17.

$$
\int_{-\infty}^{\infty} \frac{\left|\alpha_{s}+\beta_{s}-\pi\right| d s}{\cosh ^{2}(b l(s) / 2)}<\frac{2 \pi^{2}}{b}
$$

Proof. Since $\left|\alpha_{s}-\beta_{s}\right|<\pi$, the 1st variation formula acquires the form

$$
\frac{l^{\prime}(s)}{2 \cos \left(\left(\alpha_{s}-\beta_{s}\right) / 2\right)}=-\cos \frac{\alpha_{s}+\beta_{s}}{2}=\sin \frac{\alpha_{s}+\beta_{s}-\pi}{2} \quad \forall s \in \mathbb{R} .
$$

Here, $\cos \frac{\alpha_{s}-\beta_{s}}{2}$ becomes minimal, when $\left|\alpha_{s}-\beta_{s}\right|$ is maximal; meanwhile due to Lem. $15 \arccos \tanh (b l(s) / 2) \leq \alpha_{s}, \beta_{s} \leq \pi-\arccos \tanh (b l(s) / 2)$ and so

$$
\begin{aligned}
\cos \frac{\alpha_{s}-\beta_{s}}{2} & \geq \cos \frac{\pi-2 \arccos \tanh (b l(s) / 2)}{2}=\sin \arccos \tanh (b l(s) / 2) \\
& =\sqrt{1-\tanh ^{2}(b l(s) / 2)}=1 / \cosh (b l(s) / 2)
\end{aligned}
$$


Now if $l^{\prime} \geq 0$ on $[q, r]$ then

$$
\begin{aligned}
0 \leq \alpha_{s}+\beta_{s}-\pi & \leq \pi \sin \frac{\alpha_{s}+\beta_{s}-\pi}{2}=\frac{\pi l^{\prime}(s)}{2 \cos \left(\left(\alpha_{s}-\beta_{s}\right) / 2\right)} \\
& \leq \frac{\pi l^{\prime}(s) \cosh (b l(s) / 2)}{2} \quad \forall q \leq s \leq r \\
\Rightarrow \quad \int_{q}^{r} \frac{\left|\alpha_{s}+\beta_{s}-\pi\right| d s}{\cosh ^{2}(b l(s) / 2)} & \leq \int_{q}^{r} \frac{\pi l^{\prime}(s) d s}{2 \cosh (b l(s) / 2)}=\int_{l(q)}^{l(r)} \frac{\pi d l}{2 \cosh (b l / 2)} \\
& =\left.\frac{2 \pi \arctan \mathrm{e}^{b l(s) / 2}}{b}\right|_{q} ^{r}
\end{aligned}
$$

just as computed in the proof of Cor. 16. In case that $l^{\prime} \leq 0$ on $[q, r]$, deduce analogously

$$
\begin{gathered}
0 \geq \alpha_{s}+\beta_{s}-\pi \geq \pi \sin \frac{\alpha_{s}+\beta_{s}-\pi}{2} \geq \frac{\pi l^{\prime}(s) \cosh (b l(s) / 2)}{2} \\
\Rightarrow \quad \int_{q}^{r} \frac{\left|\alpha_{s}+\beta_{s}-\pi\right| d s}{\cosh ^{2}(b l(s) / 2)} \leq \int_{q}^{r} \frac{-\pi l^{\prime}(s) d s}{2 \cosh (b l(s) / 2)}=\left.\frac{-2 \pi \arctan \mathrm{e}^{b l(s) / 2}}{b}\right|_{q} ^{r} .
\end{gathered}
$$

In light of Rem. 11,

$$
\int_{-\infty}^{\infty} \frac{\left|\alpha_{s}+\beta_{s}-\pi\right| d s}{\cosh ^{2}(b l(s) / 2)} \leq\left.\frac{2 \pi \arctan \mathrm{e}^{b l(s) / 2}}{b}\right|_{0} ^{\infty}-\left.\frac{2 \pi \arctan \mathrm{e}^{b l(s) / 2}}{b}\right|_{-\infty} ^{0}<\frac{2 \pi^{2}}{b}
$$

if $C$ contains closed geodesics; while for cylinders without closed geodesics even

$$
\int_{-\infty}^{\infty} \frac{\left|\alpha_{s}+\beta_{s}-\pi\right| d s}{\cosh ^{2}(b l(s) / 2)} \leq\left.\frac{2 \pi \arctan \mathrm{e}^{b l(s) / 2}}{b}\right|_{-\infty} ^{\infty}<\frac{\pi^{2}}{b}
$$

holds.

THEOREM 18. Let $C$ be a cylinder free of conjugate points and $K \geq-b^{2}$. If $\lim \sup _{s \rightarrow \pm \infty} \frac{l(s)}{\ln |s|}<1 / b$, then $C$ is flat.

Proof. For $r \geq 0$ define $L(r):=\max (l(r), l(-r))$ and $W(r):=\int_{-r}^{r} V(s) d s$. Using Cor. 16, Lem. 14 states

$$
\begin{aligned}
W^{2}(r) & \leq 32 \pi\left(V(-r) \int_{0}^{l(-r)} \frac{d t}{y_{-r}(t)}+V(r) \int_{0}^{l(r)} \frac{d t}{y_{r}(t)}\right)+8 \pi^{2}\left(\alpha_{r}+\beta_{r}-\alpha_{-r}-\beta_{-r}\right)^{2} \\
& \leq \frac{32 \pi^{2} \cosh ^{2}(b L(r) / 2)}{b}(V(-r)+V(r))+8 \pi^{2}\left(\alpha_{r}+\beta_{r}-\alpha_{-r}-\beta_{-r}\right)^{2} .
\end{aligned}
$$

The triangle-inequality yields

$$
\begin{aligned}
\left(\alpha_{r}+\beta_{r}-\alpha_{-r}-\beta_{-r}\right)^{2} & \leq 2 \pi\left|\alpha_{r}+\beta_{r}-\alpha_{-r}-\beta_{-r}\right| \\
& \leq 2 \pi\left(\left|\alpha_{r}+\beta_{r}-\pi\right|+\left|\alpha_{-r}+\beta_{-r}-\pi\right|\right) \\
& \leq 2 \pi\left(\frac{\left|\alpha_{r}+\beta_{r}-\pi\right|}{\cosh ^{2}(b l(r) / 2)}+\frac{\left|\alpha_{-r}+\beta_{-r}-\pi\right|}{\cosh ^{2}(b l(-r) / 2)}\right) \cosh ^{2} \frac{b L(r)}{2}
\end{aligned}
$$


- which together with $V(-r)+V(r)=W^{\prime}(r)$ implies

$$
W^{2}(r) \leq \frac{32 \pi^{2}}{b}\left(W^{\prime}(r)+\frac{b \pi\left|\alpha_{r}+\beta_{r}-\pi\right|}{2 \cosh ^{2}(b l(r) / 2)}+\frac{b \pi\left|\alpha_{-r}+\beta_{-r}-\pi\right|}{2 \cosh ^{2}(b l(-r) / 2)}\right) \cosh ^{2} \frac{b L(r)}{2} .
$$

Now assume that $W(R)>0$ for some $R>0$ - so by the monotonicity of $W$ also $W(r)>0 \forall r \geq R$. Then

$$
\frac{W^{\prime}(r)}{W^{2}(r)} \geq \frac{b}{32 \pi^{2} \cosh ^{2}(b L(r) / 2)}-\frac{b \pi}{2 W^{2}(r)}\left(\frac{\left|\alpha_{r}+\beta_{r}-\pi\right|}{\cosh ^{2}(b l(r) / 2)}+\frac{\left|\alpha_{-r}+\beta_{-r}-\pi\right|}{\cosh ^{2}(b l(-r) / 2)}\right)
$$

for all $r \geq R$; and integration leads to (cf. [BK], Lem 3.12)

$$
\begin{aligned}
\frac{1}{W(R)} \geq & \left.\frac{-1}{W(r)}\right|_{R} ^{\infty}=\int_{R}^{\infty} \frac{W^{\prime}(r) d r}{W^{2}(r)} \\
\geq & \int_{R}^{\infty} \frac{b d r}{32 \pi^{2} \cosh ^{2}(b L(r) / 2)} \\
& -\frac{b \pi}{2 W^{2}(R)} \int_{R}^{\infty}\left(\frac{\left|\alpha_{r}+\beta_{r}-\pi\right|}{\cosh ^{2}(b l(r) / 2)}+\frac{\left|\alpha_{-r}+\beta_{-r}-\pi\right|}{\cosh ^{2}(b l(-r) / 2)}\right) d r \\
\geq & \int_{R}^{\infty} \frac{b d r}{32 \pi^{2} \cosh ^{2}(b L(r) / 2)}-\frac{b \pi}{2 W^{2}(R)} \int_{-\infty}^{\infty} \frac{\left|\alpha_{s}+\beta_{s}-\pi\right| d s}{\cosh ^{2}(b l(s) / 2)} \\
> & \int_{R}^{\infty} \frac{b d r}{32 \pi^{2} \cosh ^{2}(b L(r) / 2)}-\frac{\pi^{3}}{W^{2}(R)}
\end{aligned}
$$

according Lem. 17.

But by the assumption, $b L(r)<\ln r$ for $r>R, R$ sufficiently large, so

$$
\int_{R}^{\infty} \frac{d r}{\cosh ^{2}(b L(r) / 2)}>\int_{R}^{\infty} \frac{4 d r}{\mathrm{e}^{b L(r)}+3} \geq \int_{R}^{\infty} \frac{4 d r}{r+3}=\infty
$$

- a contradiction. So $W \equiv 0$. Using Lem. 12, this proves

$$
\int_{S C} U^{2}(v) d \mu(v)=\int_{-\infty}^{\infty} V(s) d s=\limsup _{r \rightarrow \infty} W(r)=0 .
$$

Hence $U=0 \mu$-a.e. and therefore $K \equiv 0$ by Riccati equation, as $\mathrm{K}$ is continuous.

\section{REFERENCES}

[B] K. Burns, The flat strip theorem fails for surfaces with no conjugate points, Proc. Amer. Math. Soc., 115 (1992), pp. 199-206.

[BBB] W. Ballmann, M. Brin and K. Burns, On surfaces with no conjugate points, J. Differential Geometry, 25 (1987), pp. 249-273.

[BI] D. Burago And S. Ivanov, Riemannian tori without conjugate points are flat Geom. Funct. Anal., 4 (1995), pp. 259-269.

[BK] K. Burns And G. KnIEPER, Rigidity of surfaces with no conjugate points, J. Differential Geometry, 34 (1991), pp. 623-650.

[CK] C. B. CRoke AND B. KleIner, A rigidity theorem for manifolds without conjugate points, Ergod. Th. Dyn. Sys., 18 (1998), pp. 813-829.

[Gr] L. W. Green, Surfaces without conjugate points, Trans. Amer. Math. Soc., 76 (1954), pp. 529-546.

[Gu] R. Gulliver, On the variety of manifolds without conjugate points, Trans. Amer. Math. Soc., 210 (1975), pp. 185-201. 
[H] E. Hopf, Closed surfaces without conjugate points, Proc. Nat. Acad. Sci., 34 (1948), pp. 4751.

[I] N. Innami, Euclidean metric and flat metric outside a compact set, Proc. Amer. Math. Soc., 105 (1989), pp. 701-705.

[MZ] S. MENDONCA AND D. ZHou, Maximal ergodic theorems and applications to Riemannian geometry, Isr. J. Math., 139 (2004), pp. 319-335. 
H. KOEHLER 